Conclusions Both CT MAMEF assays demonstrated moderate sensitivity and high specificity when using dry-shipped vaginal swabs. The cryptic plasmid assay was more sensitive than the $16 \mathrm{~S}$ rRNA assay. MAMEF is an ultra-rapid platform with results in less than 10 minutes making it ideal as a point-of-care test.

\section{P5.062 AN EASY TO USE REAL TIME PCR TEST FOR CHLAMYDIA TRACHOMATIS AND NEISSERIA GONORRHOEAE PROVIDING RESULTS THAT CAN GUIDE TREATMENT CHOICES BEFORE THE PATIENT LEAVES THE CLINIC}

doi:10.1136/sextrans-2013-051184.1106

'C A Gaydos, ${ }^{2 B}$ Van Der Pol, ' $\mathrm{M}$ Jett-Goheen, ${ }^{1} \mathrm{M}$ Barnes, ${ }^{1} \mathrm{~N}$ Quinn, ${ }^{3} \mathrm{C}$ Clark, ${ }^{3} \mathrm{G} \mathrm{E}$ Daniel, ${ }^{3 P}$ B Dixon, E W Hook, '3II. 'Johns Hopkins University, Baltimore, MD, United States: 2Indiana Univ School of Public Health \& School of Med, Bloomington and Indianapolis, IN, United States; ${ }^{3}$ University of Alabama at Birmingham, Birmingham, AL, United States

Background New diagnostics for Chlamydia trachomatis (CT) and Neisseria gonorrhoeae (NG) that can provide accurate, rapid, and timely results are urgently needed so that patients can be treated at the time they visit a clinic. The Cepheid GeneXpert ${ }^{\circledR}$ (Xpert) CT/ NG assay is a rapid nucleic acid amplified test (NAAT) assay that can be performed in on-site laboratories. The assay detects DNA of CT and NG from female endocervical, patient-collected vaginal, and urine specimens, and in male urine from symptomatic and asymptomatic individuals.

Methods The Xpert test is a modular, cartridge-based, "walkaway" platform for testing which requires no manipulation from specimen loading until results are available (90 minutes). We compared results from the Xpert assay to results from two currently approved nucleic acid amplification assays (Aptima Combo2 and ProbeTec) in 1,722 females and 1,387 males. Results included a specimen adequacy control result and an amplification control result. The targets were two highly conserved, non-contiguous NG-unique chromosomal targets for NG and a chromosomal CT target.

Results Compared to patient-infected-status (PIS), the Xpert results for chlamydia demonstrated sensitivities for endocervical, vaginal, and urine samples of $97.4 \%, 98.7 \%$, and $97.6 \%$, respectively, in females and in male urine a sensitivity of $97.5 \%$. All specificity estimates were $\geq 99.4 \%$. Results for gonorrhoea demonstrated sensitivities for endocervical, vaginal, and urine samples of $100.0 \%$, $100.0 \%$, and $95.6 \%$, respectively, in females, with specificities > $99.9 \%$. In male urine, the sensitivity for gonorrhoea was $97.50 \%$ and the specificity was $99.9 \%$.

Conclusions The GeneXpert ${ }^{\circledR}$ System is a closed, self-contained, fully-integrated, automated platform for CT/NG testing that demonstrated excellent sensitivities and specificities in women and men. This relatively rapid short-turn-around-time test can provide results to guide treatment decisions before patients leave the clinical setting. Such rapid treatment could potentially improve chlamydia and gonorrhoea control efforts.

\section{P5.063 INFLUENCE OF TEMPERATURE, MEDIUM AND STORAGE DURATION ON CHLAMYDIA TRACHOMATIS DNA DETECTION BY POLYMERASE CHAIN REACTION}

doi:10.1136/sextrans-2013-051184.1107

1,2L van Dommelen, 1,2P F G Wolffs, ${ }^{1,2}$ F H van Tiel, ${ }^{3,1,2}$ N Dukers, ' $\mathrm{S}$ B Herngreen, ${ }^{1,2} \mathrm{C}$ A Bruggeman, ${ }^{3,1,2} \mathrm{C}$ J P A Hoebe. 'Maastricht University Medical Centre, Maastricht, The Netherlands; ${ }^{2}$ CAPHRI School of Public Health and Primary Care, Maastricht, The Netherlands; ${ }^{3}$ South Limburg Public Health Service, Geleen, The Netherlands

Background Chlamydia trachomatis $(\mathrm{Ct})$ is the most prevalent bacterial sexually transmitted microorganism worldwide. Many researchers conveniently use stored samples for $\mathrm{Ct}$ research. We assessed the impact of four different temperature conditions, six different types of medium and five increasing lengths of duration of storage, on Ct DNA detection.

Methods Phosphate buffered saline (PBS), 2-sucrose-phosphate (2-SP) medium, COBAS Amplicor medium and urine samples were spiked with the same amount of $\mathrm{Ct}$ serovar $\mathrm{D}$ elementary bodies and were stored in at room temperature (RT), $4^{\circ} \mathrm{C},-20^{\circ} \mathrm{C}$ and $-80^{\circ} \mathrm{C}$. Clinical $\mathrm{Ct}$ positive urine samples and $\mathrm{Ct}$ positive swabs in COBAS Amplicor medium were collected, pooled and stored at the same 4 temperatures. Samples (136 clinical and 287 spiked samples) were tested in triplicate on day 0 and subsequently after 1, 7,14 and 30 days of storage and two years hereafter for the presence of Ct DNA. Approximately 3000 plasmids were available per PCR reaction and each sample was thawed only once. Cycle thresholds were analysed using generalised linear models, controlling for repeated measurements.

Results Ct could be detected in all clinical samples and spiked media and cycle thresholds were stable over time with few exceptions. For Ct DNA detection in spiked COBAS Amplicor medium, cycle thresholds increased within the first month at $-20^{\circ} \mathrm{C}$ and $-80^{\circ} \mathrm{C}$ (both $\mathrm{p}<0.01$ ), but decreased in the samples frozen after two years of storage. In spiked urine and pooled clinical urine samples, the cycle threshold decreased within the first month $(p<0.01)$, including all but one $\left(4^{\circ} \mathrm{C}, \mathrm{p}=0.09\right)$ of the studied temperatures, but increased after two years in the frozen samples.

Conclusion Our results demonstrate that storage conditions and duration hardly affect Ct DNA detection by PCR in a negative manner, although frozen urine samples, stored for prolonged periods (more than two years), could become Ct negative.

\section{P5.064 PRELIMINARY ANALYTICAL EVALUATION OF ARTUS $®$ CT/NG, FOR SIMULTANEOUS DETECTION OF NEISSERIA GONORRHOEAE AND CHLAMYDIA TRACHOMATIS}

doi:10.1136/sextrans-2013-051184.1108

1,2S N Tabrizi, ${ }^{1} \mathrm{~J}$ Twin, ${ }^{3} \mathrm{M}$ Unemo, ${ }^{4} \mathrm{~A}$ Wiezer, ${ }^{5} \mathrm{~A}$ Limnios, ${ }^{5} \mathrm{M}$ Lahra, ${ }^{1,6} \mathrm{~S}$ Garland. ${ }^{1}$ The Royal Women's Hospital, Parkville, Australia; 'Department of Obstetrics and Gynaecology, University of Melbourne, Parkville, Australia; 'WHO Collaborating Centre for Gonorrhoea and other STIS, Department of Laboratory Medicine, Microbiology, Örebro University Hospital, Örebro, Sweden; ${ }^{4}$ OIAGEN Research and Development, Hamburg, Germany; ${ }^{5}$ WHO Collaborating Centre for STD, Microbiology Department, The Prince of UK Hospital, Randwick, Australia; ${ }^{62)}$ Department of Obstetrics and Gynaecology, University of Melbourne, Parkville, Australia

Introduction Currently there are a number of molecular amplification assays for detection of Neisseria gonorrhoeae and Chlamydia trachomatis. Prior to introduction of any new assay, it should be thoroughly evaluated for potential false positive and false negative results due to cross reaction with other species, and potential mutations and genetic exchanges with other closely related organisms. Artus ${ }^{\circledR}$ CT/NG is currently a research use only kit (with the fully automated version to be released later this year)for simultaneously detection of $C$. trachomatis and $N$. gonorrhoeae. We performed preliminary analytical evaluation of this assay.

Method This study evaluated the Artus ${ }^{\circledR}$ CT/NG assay with 290 characterised culture isolates obtained from the Neisseria Reference Laboratory at the World Health Organization Collaborating Centres for Sexually Transmitted Disease (STD) in Sydney and WHO Collaborating Centre for Gonorrhoea and other STIs in Örebro, Sweden. Strains included 148 N. gonorrhoeae isolates, 130 isolates of non-gonococcal Neisseria species, 12 isolates of other species closely related to Neisseria and 16 C. trachomatis strains of different serovars (including LGV and nvCT strains).

Results All C. trachomatis and N. gonorrhoeae isolates were detected. A detection sensitivity of 10 genome copies per reaction was obtained with all C. trachomatis serovars as well as a representative $N$. gonorrhoeae control strain. All 142 non-gonococcal isolates were negative on the assay. 